\title{
Article \\ Central and Local Limit Theorems for Numbers of the Tribonacci Triangle
}

\author{
Igoris Belovas $\mathbb{D}$
}

check for updates

Citation: Belovas, I. Central and Local Limit Theorems for Numbers of the Tribonacci Triangle. Mathematics 2021, 9, 880. https://doi.org/ $10.3390 /$ math 9080880

Academic Editor: Lev Klebanov

Received: 17 March 2021

Accepted: 14 April 2021

Published: 16 April 2021

Publisher's Note: MDPI stays neutral with regard to jurisdictional claims in published maps and institutional affiliations.

Copyright: (C) 2021 by the author. Licensee MDPI, Basel, Switzerland. This article is an open access article distributed under the terms and conditions of the Creative Commons Attribution (CC BY) license (https:// creativecommons.org/licenses/by/ $4.0 /)$.
Institute of Data Science and Digital Technologies, Vilnius University, LT-04812 Vilnius, Lithuania; Igoris.Belovas@mif.vu.lt

Abstract: In this research, we continue studying limit theorems for combinatorial numbers satisfying a class of triangular arrays. Using the general results of Hwang and Bender, we obtain a constructive proof of the central limit theorem, specifying the rate of convergence to the limiting (normal) distribution, as well as a new proof of the local limit theorem for the numbers of the tribonacci triangle.

Keywords: tribonacci matrix; triangular array; limit theorems; rate of convergence; generating functions

MSC: 05A15; 39A06; 60F05

\section{Introduction}

The tribonacci triangle (also known as the Delannoy triangle [1], cf. OEIS Number sequence A008288 [2]) was introduced to derive the expansion of the tribonacci numbers (OEIS Number sequence A000073 [3]). The sum of the elements along a rising diagonal of this triangle (see Table 1 ) equals the tribonacci number: $1,1,2,4,7,13,24, \ldots$.

Table 1. The tribonacci triangle ( $T_{n, k}$ numbers, see Equation (1)).

\begin{tabular}{cccccccc}
\hline $\mathrm{n}$ & 0 & 1 & 2 & 3 & 4 & 5 & $\ldots$ \\
\hline 0 & 1 & 0 & 0 & 0 & 0 & 0 & $\ldots$ \\
1 & 1 & 1 & 0 & 0 & 0 & 0 & $\ldots$ \\
2 & 1 & 3 & 1 & 0 & 0 & 0 & $\ldots$ \\
3 & 1 & 5 & 5 & 1 & 0 & 0 & $\ldots$ \\
4 & 1 & 7 & 13 & 7 & 1 & 0 & $\ldots$ \\
5 & 1 & 9 & 25 & 25 & 9 & 1 & $\ldots$ \\
$\ldots$ & $\ldots$ & $\ldots$ & $\ldots$ & $\ldots$ & $\ldots$ & $\ldots$ & $\ldots$ \\
\hline
\end{tabular}

Numbers satisfying the triangle can be defined by the following recurrent expression:

$$
T_{n, k}= \begin{cases}1, & \text { for } k=0 \text { or } k=n, \\ 0, & \text { for } n<k \text { or } n<0 \text { or } k<0 \\ T_{n-1, k-1}+T_{n-1, k}+T_{n-2, k-1}, & \text { otherwise }\end{cases}
$$

Barry [4] showed that the closed form for numbers satisfying the tribonacci triangle is

$$
T_{n, k}=\sum_{j=0}^{\min (k, n-k)} C_{k}^{j} C_{n-j}^{k}
$$


The tribonacci (Delannoy)-like triangles and their generalizations are intensively examined nowadays. Amrouche, Belbachir and Ramírez [1,5] have studied the unimodality of sequences located in the triangle's infinite transversals and derived the explicit formulation of the linear recurrence sequence satisfied by the sum of the elements lying over any finite ray of the generalized tribonacci matrix. Kuhapatanakul [6] has examined the connection between a generalized tribonacci triangle and a generalized Fibonacci sequence. The total positivity of Delannoy-like triangles has been considered by $\mathrm{Mu}$ and Zheng [7]. Yang, Zheng and Yuan [8] have studied the inverses of the generalized Delannoy matrices.

The present research extends the investigations of the asymptotics for Delannoy numbers undertaken by Noble [9,10] and Wang, Zheng and Chen [11] (as well as our research into central and local limit theorems for combinatorial numbers satisfying a class of triangular arrays [12-15]). Noble has obtained asymptotic expansions for the central weighted Delannoy numbers $\left(u_{r, r}\right)$ and the numbers along the the diagonal with slope 2 $\left(u_{r, 2 r}\right)$. Wang, Zheng and Chen showed the asymptotic normality of Delannoy numbers, using the properties of the zeroes of Delannoy polynomials. In this work we provide a new constructive proof of the central limit theorem for the numbers of the tribonacci triangle, specifying the rate of convergence to the limiting distribution (in the process we receive the closed exact expression for the variance of the random variable, associated to the numbers of the tribonacci triangle, missing in the work of Wang et al.), together with a new proof of the local limit theorem.

The paper is organized in the following way. The first part is the introduction. In the second part, we specify the moment-generating function of the numbers of the tribonacci triangle and calculate exact expressions for the expectation and the variance of the random variable, associated to the numbers of the tribonacci triangle. The third and fourth sections are devoted to the central and local limit theorems.

Throughout this paper, we denote by $\Phi_{\mu, \sigma}(x)$ the cumulative distribution function of the normal distribution with the mean $\mu$ and the standard deviation $\sigma$; by $\varphi_{\mu, \sigma}(x)$ we denote the corresponding density function.

All limits in the paper, unless specified, are taken as $n \rightarrow \infty$.

\section{Moment-Generating Function}

Let us consider the generating function of the numbers given by Equation (1),

$$
G(x, y)=\sum_{n=0}^{\infty} \sum_{k=0}^{\infty} T_{n, k} x^{n} y^{k}=\sum_{n=0}^{\infty} \sum_{k=0}^{n} T_{n, k} x^{n} y^{k} .
$$

Lemma 1. (Alladi and Hoggatt [16]). The bivariate generating function of the numbers of the tribonacci triangle is

$$
G(x, y)=\frac{1}{1-x-x y-x^{2} y} .
$$

Let $A_{n}$ be an integral random variable with the probability mass function

$$
P\left(A_{n}=k\right)=\frac{T_{n, k}}{\sum_{k=0}^{n} T_{n, k}} .
$$

The moment-generating function of the random variable $A_{n}$ equals

$$
M_{n}(s)=E\left(e^{A_{n} s}\right)=\sum_{k=0}^{n} P\left(A_{n}=k\right) e^{k s}=S_{n}^{-1} \sum_{k=0}^{n} T_{n, k} e^{k s},
$$

where $S_{n}$ stands for the sum of the $n$-th row of the triangle,

$$
S_{n}=\sum_{k=0}^{n} T_{n, k}
$$


Combining the definition of the generating function from Equations (2) and (5), we obtain

$$
G\left(x, e^{s}\right)=\sum_{n=0}^{\infty} x^{n} \sum_{k=0}^{n} T_{n, k} e^{s k}=\sum_{n=0}^{\infty} x^{n} S_{n} M_{n}(s) .
$$

Thus, the partial differentiation of the generating function $G(x, y)$ at $x=0$, yields us the moment-generating function

$$
M_{n}(s)=\left.\frac{1}{S_{n} n !} \frac{\partial^{n}}{\partial x^{n}} G\left(x, e^{s}\right)\right|_{x=0} .
$$

Note that, since $M_{n}(0)=1$, we have a formula for the sum of the $n$-th row,

$$
S_{n}=\left.\frac{1}{n !} \frac{\partial^{n}}{\partial x^{n}} G(x, 1)\right|_{x=0} .
$$

Lemma 2. The moment-generating function of the random variable $A_{n}$, associated to the numbers of the tribonacci triangle given by Equation (4), is

$$
M_{n}(s)=\frac{\sqrt{8}}{\theta(s)} \frac{\left(1+e^{s}+\theta(s)\right)^{n+1}-\left(1+e^{s}-\theta(s)\right)^{n+1}}{(2+\sqrt{8})^{n+1}-(2-\sqrt{8})^{n+1}},
$$

here

$$
\theta(s)=\sqrt{e^{2 s}+6 e^{s}+1}, \quad s \in \mathbb{R} .
$$

Proof. Let us consider the denominator of $G\left(x, e^{s}\right)$ from Equation (3),

$$
1-\left(1+e^{s}\right) x-e^{s} x^{2}=-e^{s}\left(x^{2}+\left(1+e^{-s}\right) x-e^{-s}\right)=-e^{s}\left(x-x_{1}\right)\left(x-x_{2}\right),
$$

where

$$
x_{1}=r_{1}(s)=-\frac{\left(1+e^{-s}\right)+e^{-s} \theta(s)}{2}, \quad x_{2}=r_{2}(s)=-\frac{\left(1+e^{-s}\right)-e^{-s} \theta(s)}{2} .
$$

Next,

$$
\begin{aligned}
& \frac{\partial^{n}}{\partial x^{n}}\left[\frac{1}{\left(x-x_{1}\right)\left(x-x_{2}\right)}\right]_{x=0}=\frac{1}{x_{1}-x_{2}}\left[\frac{\partial^{n}}{\partial x^{n}}\left(\frac{1}{x-x_{1}}\right)-\frac{\partial^{n}}{\partial x^{n}}\left(\frac{1}{x-x_{2}}\right)\right]_{x=0} \\
& =\frac{(-1)^{n} n !}{x_{1}-x_{2}}\left[\frac{1}{\left(x-x_{1}\right)^{n+1}}-\frac{1}{\left(x-x_{2}\right)^{n+1}}\right]_{x=0}=\frac{n !}{x_{2}-x_{1}} \frac{x_{2}^{n+1}-x_{1}^{n+1}}{\left(x_{1} x_{2}\right)^{n+1}} .
\end{aligned}
$$

Now, using Equation (11), we calculate the $n$-th derivative of $G\left(x, e^{s}\right)$,

$$
\left.\frac{\partial^{n}}{\partial x^{n}} G\left(x, e^{s}\right)\right|_{x=0}=n ! \frac{\left(1+e^{s}+\theta(s)\right)^{n+1}-\left(1+e^{s}-\theta(s)\right)^{n+1}}{2^{n+1} \theta(s)} .
$$

Thus, by Equation (7),

$$
S_{n}=\frac{(2+\sqrt{8})^{n+1}-(2-\sqrt{8})^{n+1}}{2^{n+1} \sqrt{8}},
$$

yielding us the statement of the lemma (cf. Equation (6)). 
Lemma 3. The expectation and the variance of the random variable $A_{n}$, associated to the numbers of the tribonacci triangle from Equation (4), are

$$
\begin{aligned}
\mu_{n} & =\frac{n}{2} \\
\sigma_{n}^{2} & =\frac{n \sqrt{2}}{8}+\frac{\sqrt{2}-1}{8}
\end{aligned}
$$

respectively.

Proof. By Equation (8), the first and second derivatives of the moment-generating function are

$$
\begin{aligned}
M_{n}^{\prime}(s) & =\frac{\left(1+e^{s}+\theta(s)\right)^{n}\left(e^{s}+\theta^{\prime}(s)\right)-\left(1+e^{s}-\theta(s)\right)^{n}\left(e^{s}-\theta^{\prime}(s)\right)}{(\sqrt{8}(n+1))^{-1}\left((2+\sqrt{8})^{n+1}-(2-\sqrt{8})^{n+1}\right) \theta(s)} \\
& -\frac{\sqrt{8}}{\theta^{2}(s)} \frac{\left(\left(1+e^{s}+\theta(s)\right)^{n+1}-\left(1+e^{s}-\theta(s)\right)^{n+1}\right) \theta^{\prime}(s)}{(2+\sqrt{8})^{n+1}-(2-\sqrt{8})^{n+1}}
\end{aligned}
$$

and

$$
\begin{aligned}
M_{n}^{\prime \prime}(s) & =\frac{n\left(1+e^{s}+\theta(s)\right)^{n-1}\left(e^{s}+\theta^{\prime}(s)\right)^{2}+\left(1+e^{s}+\theta(s)\right)^{n}\left(e^{s}+\theta^{\prime \prime}(s)\right)}{(\sqrt{8}(n+1))^{-1}\left((2+\sqrt{8})^{n+1}-(2-\sqrt{8})^{n+1}\right) \theta(s)} \\
& -\frac{\left(\left(1+e^{s}+\theta(s)\right)^{n}\left(e^{s}+\theta^{\prime}(s)\right)-\left(1+e^{s}-\theta(s)\right)^{n}\left(e^{s}-\theta^{\prime}(s)\right)\right) \theta^{\prime}(s)}{(\sqrt{8}(n+1))^{-1}\left((2+\sqrt{8})^{n+1}-(2-\sqrt{8})^{n+1}\right) \theta^{2}(s)} \\
& -\frac{n\left(1+e^{s}-\theta(s)\right)^{n-1}\left(e^{s}-\theta^{\prime}(s)\right)^{2}+\left(1+e^{s}-\theta(s)\right)^{n}\left(e^{s}-\theta^{\prime \prime}(s)\right)}{(\sqrt{8}(n+1))^{-1}\left((2+\sqrt{8})^{n+1}-(2-\sqrt{8})^{n+1}\right) \theta(s)} \\
& -\frac{(n+1)\left(1+e^{s}+\theta(s)\right)^{n}\left(e^{s}+\theta^{\prime}(s)\right) \theta^{\prime}(s)+\left(1+e^{s}+\theta(s)\right)^{n+1} \theta^{\prime \prime}(s)}{(\sqrt{8})^{-1}\left((2+\sqrt{8})^{n+1}-(2-\sqrt{8})^{n+1}\right) \theta^{2}(s)} \\
& +\frac{2\left(1+e^{s}+\theta(s)\right)^{n+1}\left(\theta^{\prime}(s)\right)^{2}-2\left(1+e^{s}-\theta(s)\right)^{n+1}\left(\theta^{\prime}(s)\right)^{2}}{(\sqrt{8})^{-1}\left((2+\sqrt{8})^{n+1}-(2-\sqrt{8})^{n+1}\right) \theta^{3}(s)} \\
& +\frac{(n+1)\left(1+e^{s}-\theta(s)\right)^{n}\left(e^{s}-\theta^{\prime}(s)\right) \theta^{\prime}(s)+\left(1+e^{s}-\theta(s)\right)^{n+1} \theta^{\prime \prime}(s)}{(\sqrt{8})^{-1}\left((2+\sqrt{8})^{n+1}-(2-\sqrt{8})^{n+1}\right) \theta^{2}(s)}
\end{aligned}
$$

Note that (cf. Equation (9))

$$
\begin{aligned}
& \theta^{\prime}(s)=\frac{e^{2 s}+3 e^{s}}{\left(e^{2 s}+6 e^{s}+1\right)^{1 / 2}}=\frac{e^{2 s}+3 e^{s}}{\theta(s)} \\
& \theta^{\prime \prime}(s)=\frac{e^{4 s}+9 e^{3 s}+11 e^{2 s}+3 e^{s}}{\left(e^{2 s}+6 e^{s}+1\right)^{3 / 2}}=\frac{e^{4 s}+9 e^{3 s}+11 e^{2 s}+3 e^{s}}{\theta^{3}(s)},
\end{aligned}
$$

hence,

$$
\begin{aligned}
& \theta^{\prime}(0)=\sqrt{2}, \\
& \theta^{\prime \prime}(0)=\frac{3 \sqrt{2}}{4} .
\end{aligned}
$$

Next, calculating the expectation, by Equations (12) and (14), we obtain

$$
\begin{aligned}
\mu_{n} & =M_{n}^{\prime}(0)=(n+1) \frac{(2+\sqrt{8})^{n}(1+\sqrt{2})-(2-\sqrt{8})^{n}(1-\sqrt{2})}{(2+\sqrt{8})^{n+1}-(2-\sqrt{8})^{n+1}}-\frac{1}{2} \\
& =(n+1) \frac{2^{n}(1+\sqrt{2})^{n+1}-2^{n}(1-\sqrt{2})^{n+1}}{2^{n+1}(1+\sqrt{2})^{n+1}-2^{n+1}(1-\sqrt{2})^{n+1}}-\frac{1}{2}=\frac{n}{2^{\prime}}
\end{aligned}
$$

yielding us the first statement of the lemma. 
Calculating the variance (cf. Equations (13) and (15)), we obtain

$$
\begin{aligned}
\sigma_{n}^{2} & =M_{n}^{\prime \prime}(0)-\mu_{n}^{2}=\frac{n(2+\sqrt{8})^{n-1}(1+\sqrt{2})^{2}-n(2-\sqrt{8})^{n-1}(1-\sqrt{2})^{2}}{(n+1)^{-1}\left((2+\sqrt{8})^{n+1}-(2-\sqrt{8})^{n+1}\right)} \\
& +\frac{\left.(2+\sqrt{8}))^{n}(1+3 \sqrt{2} / 4)-(2-\sqrt{8})\right)^{n}(1-3 \sqrt{2} / 4)}{(n+1)^{-1}\left((2+\sqrt{8})^{n+1}-(2-\sqrt{8})^{n+1}\right)} \\
& -\frac{\left.(2+\sqrt{8}))^{n}(1+\sqrt{2})-(2-\sqrt{8})\right)^{n}(1-\sqrt{2})}{2(n+1)^{-1}\left((2+\sqrt{8})^{n+1}-(2-\sqrt{8})^{n+1}\right)} \\
& -\frac{(n+1)(2+\sqrt{8})^{n}(1+\sqrt{2}) \sqrt{2}-(n+1)(2-\sqrt{8})^{n}(1-\sqrt{2}) \sqrt{2}}{\sqrt{8}\left((2+\sqrt{8})^{n+1}-(2-\sqrt{8})^{n+1}\right)} \\
& +\frac{1}{2}-\frac{(2+\sqrt{8})^{n+1} 3 \sqrt{2} / 4-(2-\sqrt{8})^{n+1} 3 \sqrt{2} / 4}{\sqrt{8}\left((2+\sqrt{8})^{n+1}-(2-\sqrt{8})^{n+1}\right)} \\
& =\frac{n(n+1)}{4}-\frac{n+1}{4}-\frac{n+1}{4}+\frac{1}{2}-\frac{3}{8}-\frac{n^{2}}{4} \\
& +\frac{\left.(2+\sqrt{8}))^{n}(2+\sqrt{8}+2+\sqrt{2})-(2-\sqrt{8})\right)^{n}(2-\sqrt{8}+2-\sqrt{2})}{4(n+1)^{-1}\left((2+\sqrt{8})^{n+1}-(2-\sqrt{8})^{n+1}\right)} \\
& =-\frac{n}{4}-\frac{3}{8}+\frac{n+1}{4}\left(1+\frac{1}{\sqrt{2}}\right)=\frac{n+1}{4 \sqrt{2}}-\frac{1}{8}=\frac{n \sqrt{2}}{8}+\frac{\sqrt{2}-1}{8},
\end{aligned}
$$

thus concluding the proof of the lemma.

\section{Central Limit Theorem}

Let, by Hwang [17], $\left\{\Omega_{n}\right\}$ be a sequence of integral random variables, and

$$
M_{n}(s)=e^{H_{n}(s)}\left(1+O\left(\kappa_{n}^{-1}\right)\right) .
$$

Here $O$-term is uniform for $|s| \leqslant \tau, s \in \mathbb{C}, \tau>0$, and

(i) $\quad H_{n}(s)=u(s) \phi(n)+v(s)$, with $u(s)$ and $v(s)$ analytic for $|s| \leqslant \tau$ and independent of $n, u^{\prime \prime}(0) \neq 0$;

(ii) $\phi(n) \rightarrow+\infty$;

(iii) $\quad \kappa_{n} \rightarrow+\infty$.

We apply the following Hwang's result [17] to prove the central limit theorem for the numbers of Equation (1) and specify the rate of convergence to the limiting distribution.

Theorem 1. (Hwang). Under assumptions (i)-(iii),

$$
\left|P\left(\frac{\Omega_{n}-u^{\prime}(0) \phi(n)}{\sqrt{u^{\prime \prime}(0) \phi(n)}}<x\right)-\Phi(x)\right|=O\left(\frac{1}{\kappa_{n}}+\frac{1}{\sqrt{\phi(n)}}\right),
$$

uniformly with respect to $x, x \in \mathbb{R}$.

Next we prove an auxiliary lemma.

Lemma 4. For $x \in \mathbb{R}$,

$$
-\frac{1}{3+\sqrt{8}} \leqslant \frac{1+e^{x}-\theta(x)}{1+e^{x}+\theta(x)}<0
$$

Proof. Let

$$
q(x)=\frac{1+e^{x}-\theta(x)}{1+e^{x}+\theta(x)}
$$


Calculating the first derivative, we obtain

$$
q^{\prime}(x)=\frac{2 e^{x} \theta(x)-2 \theta^{\prime}(x)-2 e^{x} \theta^{\prime}(x)}{\left(1+e^{x}+\theta(x)\right)^{2}} .
$$

Solving the equation

$$
e^{x} \theta(x)-\theta^{\prime}(x)-e^{x} \theta^{\prime}(x)=0,
$$

we get

$$
\theta^{2}(x)-e^{2 x}-4 e^{x}-3=0 \quad \Rightarrow \quad e^{x}=1,
$$

yielding us the stationary point $x_{0}=0$. Since $q^{\prime \prime}\left(x_{0}\right)=1 /(8+6 \sqrt{2})>0$, we have $\min q(x)=q\left(x_{0}\right)=-1 /(3+\sqrt{8})$, thus concluding the proof.

The following theorem shows that the numbers of the tribonacci triangle from Equation (1) are asymptotically normal, and identifies the rate of convergence to the limiting distribution.

Theorem 2. Suppose that $F_{n}(x)$ is the cumulative distribution function of the random variable $A_{n}$, then

$$
\left|F_{n}\left(\frac{x-\mu_{n}}{\sigma_{n}}\right)-\Phi(x)\right|=O\left(\frac{1}{\sqrt{n}}\right),
$$

uniformly with respect to $x, x \in \mathbb{R}$.

Proof. The logarithm of the moment-generating function equals

$$
\begin{aligned}
\log M_{n}(s) & =\underbrace{n}_{=\phi(n)} \underbrace{\log \frac{1+e^{s}+\theta(s)}{2+\sqrt{8}}}_{=u(s)}+\underbrace{\log \frac{1+e^{s}+\theta(s)}{2+\sqrt{8}}+\log \frac{\sqrt{8}}{\theta(s)}}_{=v(s)} \\
& +\underbrace{\log \left(1-\left(\frac{1+e^{s}-\theta(s)}{1+e^{s}+\theta(s)}\right)^{n+1}\right)}_{=O\left(\left(\frac{1+e^{s}-\theta(s)}{1+e^{s}+\theta(s)}\right)^{n+1}\right)} \underbrace{-\log \left(1-\left(\frac{2-\sqrt{8}}{2+\sqrt{8}}\right)^{n+1}\right)}_{=O\left(\left(\frac{2-\sqrt{8}}{2+\sqrt{8}}\right)^{n+1}\right)} .
\end{aligned}
$$

By Lemma 4 ,

$$
\left|\frac{1+e^{s}-\theta(s)}{1+e^{s}+\theta(s)}\right| \leqslant\left|\frac{2-\sqrt{8}}{2+\sqrt{8}}\right|=\frac{1}{3+\sqrt{8}}<1 .
$$

Thus,

$$
\left.M_{n}(s)=\exp (u(s) \phi(n)+v(s))\left(1+O\left(\kappa_{n}^{-1}\right)\right)\right) .
$$

Here $\kappa_{n}=(3+\sqrt{8})^{n+1}$. Note that the functions $u(s), v(s), \phi(n)$ and $\kappa_{n}$ satisfy the conditions (i)-(iii). Indeed,

$$
\begin{aligned}
& u^{\prime}(s)=\frac{e^{s}+\theta^{\prime}(s)}{1+e^{s}+\theta(s)} \\
& u^{\prime \prime}(s)=\frac{\left(e^{s}+\theta^{\prime \prime}(s)\right)\left(1+e^{s}+\theta(s)\right)-\left(e^{s}+\theta^{\prime}(s)\right)^{2}}{\left(1+e^{s}+\theta(s)^{2}\right.} .
\end{aligned}
$$

Hence,

$$
u^{\prime}(0)=\frac{1}{2}, \quad u^{\prime \prime}(0)=\frac{\sqrt{2}}{8} \neq 0,
$$

yielding us, by Equation (16), the statement of the theorem. 


\section{Local Limit Theorem}

We apply a general local limit theorem, based on the nature of the bivariate generating function of Equations (2) and (3).

Theorem 3. (Bender [18]) Let $f(z, w)$ have a power series expansion

$$
f(z, w)=\sum_{n, k \geqslant 0} u_{n, k} z^{n} w^{k}
$$

with non-negative coefficients and let $a<b$ be real numbers. Define

$$
R(\varepsilon)=\{z: a \leqslant \Re z \leqslant b, \quad|\Im z| \leqslant \varepsilon\} .
$$

Suppose there exists $\varepsilon>0, \delta>0$, a non-negative integer $m$, and functions $A(s), r(s)$ such that

(i) an $A(s)$ is continuous and non-zero for $s \in R(\varepsilon)$,

(ii) $\quad a n r(s)$ is non-zero and has a bounded third derivative for $s \in R(\varepsilon)$,

(iii) $\quad$ for $s \in R(\varepsilon)$ and $|z| \leqslant|r(s)|(1+\delta)$ function

$$
\left(1-\frac{z}{r(s)}\right)^{m} f\left(z, e^{s}\right)-\frac{A(s)}{1-z / r(s)}
$$

is analytic and bounded,

(iv) $\quad\left(r^{\prime}(\alpha) / r(\alpha)\right)^{2}-r^{\prime \prime}(\alpha) / r(\alpha) \neq 0$ for $a \leqslant \alpha \leqslant b$,

(v) $\quad f\left(z, e^{s}\right)$ is analytic and bounded for

$$
|z| \leqslant|r(\Re s)|(1+\delta), \quad \varepsilon \leqslant|\Im s| \leqslant \pi .
$$

Then we have

$$
u_{n, k} \sim \frac{n^{m} e^{-\alpha k} A(\alpha)}{m ! r^{n}(\alpha) \vartheta_{\alpha} \sqrt{2 \pi n}}
$$

uniformly for $a \leqslant \alpha \leqslant b$, where

$$
\frac{k}{n}=-\frac{r^{\prime}(\alpha)}{r(\alpha)}, \quad \vartheta_{\alpha}^{2}=\left(\frac{k}{n}\right)^{2}-\frac{r^{\prime \prime}(\alpha)}{r(\alpha)} .
$$

Now we can proceed with the local limit theorem for the numbers of the tribonacci triangle.

Theorem 4. Let

$$
\mu_{n}=\frac{n}{2}, \hat{\sigma}_{n}^{2}=\frac{n \sqrt{2}}{8},
$$

then for all $k$, such that

$$
\left|k-\mu_{n}\right|=o\left(\hat{\sigma}_{n}^{4 / 3}\right),
$$

we have the following asymptotic expression for the numbers of the tribonacci triangle in Equation (1),

$$
T_{n, k} \sim \frac{(1+\sqrt{2})^{n+1}}{2 \sqrt{2}} \varphi_{\mu_{n}, \hat{\sigma}_{n}}(k) .
$$

Proof. By Lemma 1, the generating function is

$$
f\left(z, e^{s}\right)=\frac{1}{1-z-z e^{s}-z^{2} e^{s}} .
$$


Let $r(s)$ (cf. Theorem 3) be a root of the function

$$
h\left(z, e^{s}\right)=1-z-z e^{s}-z^{2} e^{s}=-\left(e^{s} z^{2}+\left(e^{S}+1\right) z-1\right) .
$$

This function has two roots (cf. Equation (10)),

$$
z_{1}=r_{1}(s), \quad z_{2}=r_{2}(s) .
$$

Calculating derivatives, we obtain

$$
\begin{aligned}
& r_{1}^{\prime}(s)=\frac{1+\theta^{\prime}(s)-\theta(s)}{2 e^{s}}=\frac{\theta(s)-3 e^{s}-1}{2 e^{s} \theta(s)}, \\
& r_{2}^{\prime}(s)=\frac{1-\theta^{\prime}(s)+\theta(s)}{2 e^{s}}=\frac{\theta(s)+3 e^{s}+1}{2 e^{s} \theta(s)} .
\end{aligned}
$$

Note that by Theorem 1 (Bender [18]), the mean $\mu_{n}=n \mu$ and $\mu=-r^{\prime}(0) / r(0)$. Let $r(s)=r_{1}(s)$. Now we have

$$
\frac{r^{\prime}(s)}{r(s)}=\frac{\theta(s)-3 e^{s}-1}{\left(\theta(s)-e^{s}-1\right) \theta(s)}, \quad \frac{r^{\prime}(0)}{r(0)}=-\frac{1}{2},
$$

and

$$
\frac{r^{\prime \prime}(s)}{r(s)}=\frac{3 e^{3 s}+11 e^{2 s}+9 e^{s}+1-\theta^{3}(s)}{\left(\theta(s)-e^{s}-1\right) \theta^{3}(s)}, \quad \frac{r^{\prime \prime}(0)}{r(0)}=\frac{2-\sqrt{2}}{8} .
$$

Next, consider the function $A(s)$ (cf. Equation (17)) as the limit

$$
A(s)=\lim _{z \rightarrow r(s)} f\left(z, e^{s}\right)\left(1-\frac{z}{r(s)}\right)^{m+1} .
$$

Here $m+1$ is the order of the pole. Note that, if the pole is simple, then $m=0$. Now we obtain

$$
\begin{aligned}
A(s) & =-\lim _{z \rightarrow r(s)} \frac{1}{e^{s} z^{2}+\left(e^{s}+1\right) z-1}\left(1-\frac{z}{r(s)}\right) \\
& =-e^{-s} \lim _{z \rightarrow r_{1}(s)} \frac{r_{1}(s)-z}{\left(z-r_{2}(s)\right)\left(z-r_{1}(s)\right) r_{1}(s)}=\frac{1}{r(s) \theta(s)} .
\end{aligned}
$$

The function at Equation (17),

$$
\begin{aligned}
\left(1-\frac{z}{r(s)}\right)^{m} f\left(z, e^{s}\right)-\frac{A(s)}{1-z / r(s)} & =\frac{-\theta(s)+e^{s}\left(z-r_{2}(s)\right)}{e^{s}\left(z-r_{2}(s)\right)\left(z-r_{1}(s)\right) \theta(s)} \\
& =\frac{1}{\left(z-r_{2}(s)\right) \theta(s)}
\end{aligned}
$$

is analytic and bounded for

$$
|s|<\varepsilon, \quad|z|<|r(0)|+\delta=-1+\sqrt{2}+\delta .
$$

Hence, conditions (i)-(iii) and (v) of Theorem 3 are satisfied. We check the condition (iv) evaluating $\left(r^{\prime}(\alpha) / r(\alpha)\right)^{2}-r^{\prime \prime}(\alpha) / r(\alpha)$. Using Equations (21) and (22), we obtain

$$
\begin{aligned}
\left(\frac{r^{\prime}(\alpha)}{r(\alpha)}\right)^{2}-\frac{r^{\prime \prime}(\alpha)}{r(\alpha)} & =\frac{4 e^{\alpha}\left(e^{3 \alpha}+5 e^{2 \alpha}+5 e^{\alpha}+1-\theta(\alpha)\left(e^{\alpha}+1\right)^{2}\right)}{\theta^{3}(\alpha)\left(\theta(\alpha)-e^{\alpha}-1\right)^{2}} \\
& =\frac{4 e^{\alpha}\left(e^{\alpha}+1\right)\left(e^{2 \alpha}+4 e^{\alpha}+1-\theta(\alpha)\left(e^{\alpha}+1\right)\right)}{\theta^{3}(\alpha)\left(\theta(\alpha)-e^{\alpha}-1\right)^{2}}>0 .
\end{aligned}
$$


Indeed, we have

$$
e^{2 \alpha}+4 e^{\alpha}+1>\theta(\alpha)\left(e^{\alpha}+1\right)
$$

since

$$
(C+1)^{2}>\underbrace{\left(C+2 e^{\alpha}+1\right)}_{=\theta^{2}(\alpha)}\left(C-2 e^{\alpha}+1\right) .
$$

Here $C=e^{2 \alpha}+4 e^{\alpha}$.

We obtain the parameter $\alpha$ by solving the equation

$$
\frac{r^{\prime}(\alpha)}{r(\alpha)}=-\frac{k}{n} \text {. }
$$

By Equation (21), we have

$$
\begin{gathered}
\frac{1+3 e^{\alpha}-\theta(\alpha)}{\theta^{2}(\alpha)-\left(e^{\alpha}+1\right) \theta(\alpha)}=\frac{k}{n^{\prime}} \\
\left(1+3 e^{\alpha}-\left(1+6 e^{\alpha}+e^{2 \alpha}\right) \frac{k}{n}\right)^{2}=\left(1-\left(e^{\alpha}+1\right) \frac{k}{n}\right)^{2}\left(1+6 e^{\alpha}+e^{2 \alpha}\right),
\end{gathered}
$$

and

$$
2 e^{\alpha}-\left(\rho-\rho^{2}\right)\left(e^{2 \alpha}+6 e^{\alpha}+1\right)=0 .
$$

Here $\rho=k / n$. Hence, Equation (24) is equivalent to

$$
e^{2 \alpha}+2\left(3-\frac{1}{\rho-\rho^{2}}\right) e^{\alpha}+1=0 .
$$

Thus,

$$
e^{\alpha}= \begin{cases}\left(\frac{1}{\rho-\rho^{2}}-3\right)-\sqrt{\left(\frac{1}{\rho-\rho^{2}}-3\right)^{2}-1}, & k / n \leqslant 1 / 2, \\ \left(\frac{1}{\rho-\rho^{2}}-3\right)+\sqrt{\left(\frac{1}{\rho-\rho^{2}}-3\right)^{2}-1,} & k / n>1 / 2\end{cases}
$$

and

$$
\tau=e^{\alpha}=\frac{1}{\rho-\rho^{2}}-3+\frac{2 \rho-1}{\rho-\rho^{2}} \sqrt{2 \rho^{2}-2 \rho+1} .
$$

Note that $1 /\left(\rho-\rho^{2}\right) \geqslant 4$. Next, combining Equations (18), (22) and (23), we get

$$
\begin{aligned}
T_{n, k} & \sim \frac{e^{-\alpha k}}{r^{n+1}(\alpha) \theta(\alpha) \sqrt{\frac{k^{2}}{n^{2}}-\frac{3 e^{3 \alpha}+11 e^{2 \alpha}+9 e^{\alpha}+1-\theta^{3}(\alpha)}{\left(\theta(\alpha)-e^{\alpha}-1\right) \theta^{3}(\alpha)}} \sqrt{2 \pi n}} \\
& =\frac{2^{n+1} \tau^{n-k+1}}{\sqrt{2 \pi n}(\theta(\log \tau)-\tau-1)^{n+1} \sqrt{\frac{k^{2}}{n^{2}} \theta^{2}(\log \tau)-\frac{(\tau+1)^{2}}{2}-\frac{\tau^{3}+\tau^{2}-9 \tau-1}{2 \theta(\log \tau)}}} \\
& =\frac{(\sqrt{2}-1)^{-n-1}}{\sqrt[4]{2} \sqrt{2 \pi n}} \Theta_{n, k} \underbrace{\frac{(2 \sqrt{2}-2)^{n} \tau^{n-k}}{(\theta(\log \tau)-(\tau+1))^{n}}}_{=\delta_{n, k}} .
\end{aligned}
$$

Here

$$
\Theta_{n, k}=\frac{2 \sqrt[4]{2}(\sqrt{2}-1) \tau}{(\theta(\log \tau)-\tau-1) \sqrt{\frac{k^{2}}{n^{2}} \theta^{2}(\log \tau)-\frac{(\tau+1)^{2} \theta(\log \tau)+\left(\tau^{3}+\tau^{2}-9 \tau-1\right)}{2 \theta(\log \tau)}}} .
$$


Note that by Equations (19) and (20), we have

$$
\left|\frac{k}{n}-\frac{1}{2}\right|=o\left(\frac{1}{\sqrt[3]{n}}\right)
$$

hence $k / n \rightarrow 1 / 2$ and $\tau \rightarrow 1$, while $n \rightarrow \infty$. Thus, $\Theta_{n, k} \rightarrow 1$.

Assume

$$
x=\frac{k-\mu_{n}}{\hat{\sigma}_{n}} .
$$

Using Equation (19), we get

$$
\rho=\frac{k}{n}=\frac{1}{2}+\frac{x}{2 \sqrt[4]{2} \sqrt{n}} .
$$

Thus, substituting the result into Equation (26) yields

$$
|x|=o(\sqrt[6]{n}) .
$$

Next, consider the logarithm of $\delta_{n, k}$ from Equation (25),

$$
\log \delta_{n, k}=-n \log (2 \sqrt{2}-2)+\frac{n}{2}\left(1-\frac{x}{\sqrt{n} \sqrt[4]{2}}\right) \log \tau-n \log (\theta(\log \tau)-\tau-1) .
$$

Using Taylor series expansions, we obtain for large enough $n$,

$$
\begin{aligned}
\tau & =1+\frac{4 x}{\sqrt{2} \sqrt[4]{2} \sqrt{n}}+\frac{4 x^{2}}{\sqrt{2} n}+O\left(\frac{x^{3}}{n \sqrt{n}}\right), \\
\log \tau & =\frac{4 x}{\sqrt{2} \sqrt[4]{2} \sqrt{n}}+O\left(\frac{x^{3}}{n \sqrt{n}}\right), \\
\theta(\log \tau) & =2 \sqrt{2}\left(1+\frac{2 x}{\sqrt{2} \sqrt[4]{2} \sqrt{n}}+\frac{3 x^{2}}{2 \sqrt{2} n}+O\left(\frac{x^{3}}{n \sqrt{n}}\right)\right) .
\end{aligned}
$$

Substituting Equation (29) into Equation (28), we obtain

$$
\begin{aligned}
\log \delta_{n, k} & =n \log (2 \sqrt{2}-2)+\frac{n}{2}\left(1-\frac{x}{\sqrt{n} \sqrt[4]{2}}\right)\left(\frac{4 x}{\sqrt{2} \sqrt[4]{2} \sqrt{n}}+O\left(\frac{x^{3}}{n \sqrt{n}}\right)\right) \\
& -n \log \left(2 \sqrt{2}\left(1+\frac{2 x}{\sqrt{2} \sqrt[4]{2} \sqrt{n}}+\frac{3 x^{2}}{2 \sqrt{2} n}+O\left(\frac{x^{3}}{n \sqrt{n}}\right)\right)\right. \\
& \left.-1-\frac{4 x}{\sqrt{2} \sqrt[4]{2} \sqrt{n}}-\frac{4 x^{2}}{\sqrt{2} n}-O\left(\frac{x^{3}}{n \sqrt{n}}\right)-1\right) \\
& =x \sqrt[4]{2} \sqrt{n}-x^{2}+O\left(\frac{x^{3}}{\sqrt{n}}\right) \\
& -n \log \left(1+\frac{2 x}{\sqrt{2} \sqrt[4]{2} \sqrt{n}}+\frac{(3 \sqrt{2}-4) x^{2}}{(2 \sqrt{2}-2) \sqrt{2} n}+O\left(\frac{x^{3}}{n \sqrt{n}}\right)\right) .
\end{aligned}
$$

By multiplying factors and combining like terms after applying Taylor series expansion for logarithms, we obtain

$$
\log \delta_{n, k}=-\frac{x^{2}}{2}+o(1)
$$

which, combined with Equations (25) and (27), yields us the statement of the theorem.

Funding: This research received no external funding. 
Institutional Review Board Statement: Not applicable.

Informed Consent Statement: Not applicable.

Data Availability Statement: Not applicable.

Acknowledgments: The author would like to thank the anonymous reviewer for the careful reading of the manuscript and providing constructive comments and suggestions, which have helped to improve the quality of the paper.

Conflicts of Interest: The author declares no conflict of interest.

\section{References}

1. Amrouche, S.; Belbachir, H. Unimodality and linear recurrences associated with rays in the Delannoy triangle. Turk. J. Math. 2020, 44, 118-130. [CrossRef]

2. Square Array of Delannoy Numbers $D(i, j)(i \geqslant 0, j \geqslant 0)$ Read by Antidiagonals. OEIS Number Sequence A008288. The Online Encyclopedia of Integer Sequences. Available online: https:/ / oeis.org/ A008288 (accessed on 15 April 2021).

3. Tribonacci Numbers: $a(n)=a(n-1)+a(n-2)+a(n-3)$ for $n \geqslant 3$ with $a(0)=a(1)=0$ and $a(2)=1$. OEIS Number Sequence A000073. The Online Encyclopedia of Integer Sequences. Available online: https: / /oeis.org/A000073 (accessed on 15 April 2021).

4. Barry, P. On integer-sequence-based constructions of generalized Pascal triangles. J. Integer Seq. 2006, 9, 06.2.4.

5. Amrouche, S.; Belbachir, H.; Ramírez, J.L. Unimodality, linear recurrences and combinatorial properties associated to rays in the generalized Delannoy matrix. J. Differ. Equ. $201925,1200-1215$. [CrossRef]

6. Kuhapatanakul, K. Some connections between a generalized Tribonacci triangle and a generalized Fibonacci sequence. Fibonacci Q. 2012, 50, 44-50.

7. $\mathrm{Mu}, \mathrm{L} . ;$ Zheng, S. On the total positivity of Delannoy-like triangles. J. Integer Seq. 2006, 20, 17.1.6.

8. Yang, S.; Zheng, S.; Yuan, S,; He, T. Schröder matrix as inverse of Delannoy matrix. Linear Algebra Appl. 2013, 439, 3605-3614. [CrossRef]

9. Noble, R. Asymptotics of a family of binomial sums. J. Number Theory 2010, 130, 2561-2585. [CrossRef]

10. Noble, R. Asymptotics of the weighted Delannoy numbers. Int. J. Number Theory 2012, 8, 175-188. [CrossRef]

11. Wang, Y.; Zheng, S.; Chen, X. Analytic aspects of Delannoy numbers. Discrete Math. 2019, 342, 2270-2277. [CrossRef]

12. Belovas, I. A central limit theorem for coefficients of the modified Borwein method for the calculation of the Riemann zeta-function. Lith. Math. J. 2019, 59, 17-23. [CrossRef]

13. Belovas, I. A local limit theorem for coefficients of modified Borwein's method. Glas. Mat. 2019, 54, 1-9. [CrossRef]

14. Belovas, I.; Sabaliauskas, M. Series with binomial-like coefficients for the evaluation and 3D visualization of zeta functions. Informatica 2020, 31, 659-680. [CrossRef]

15. Belovas, I.; Sakalauskas, L. Limit theorems for the coefficients of the modified Borwein method for the calculation of the Riemann zeta-function values. Colloq. Math. 2019, 151, 217-227. [CrossRef]

16. Alladi, K.; Hoggatt, V.E., Jr. On Tribonacci numbers and related functions. Fibonacci Q. 1977, 15.1, 42-45.

17. Hwang, H.-K. On convergence rates in the central limit theorems for combinatorial structures. Eur. J. Comb. 1998 19, 329-343. [CrossRef]

18. Bender, E.A. Central and local limit theorems applied to asymptotic enumeration. J. Comb. Theory Ser. A 1973 15, 91-111. [CrossRef] 